\title{
The role of perceptual salience and type of instruction in children's recall of relevant and incidental dimensional values
}

\author{
RICHARD D. ODOM, JOSEPH G. CUNNINGHAM, and EILEEN ASTOR-STETSON \\ Vanderbilt University, Nashville, Tennessee 37240
}

\begin{abstract}
Kindergarten and sixth-grade children were asked to recall the array locations of dimensional values in tasks in which instructions about the relevant dimension and the salience of the task dimensions were varied. For half of the subjects within each age group, the more salient dimension was relevant and the less salient dimension was incidental to problem solution. For the remaining subjects, the procedure was reversed. Before presenting the task within each salience condition, half of the subjects were shown the values of the relevant dimension and told to remember them. The results offered no support for the position that older subjects are cognitively better able than younger subjects to select relevant and to ignore incidental information. For younger and older subjects, both relevant and incidental recall were superior for values of the more salient dimension. Prior instructions about which dimension was relevant had no effect on recall.
\end{abstract}

In a review of studies of incidental learning, Hagen and Hale (1973) concluded that as children grow older they are better able to attend selectively to central or relevant information and to ignore that which is incidental or irrelevant to solving a problem. The task in these studies typically is to recall the location of values from a relevant category or dimension. Afterward some measure is obtained of the amount of incidental information acquired while attempting to solve the initial problem. Although in most of the reviewed studies 7- to 13-year-old subjects showed improved recall with age on the initial problem-solving task, there usually were no age differences in amount of incidental recall. This difference in the age-related recall of relevant and incidental information led Hagen and Hale to their conclusions about the development of selective attention.

In contrast to these results, other research has shown that older subjects recall more incidental information than younger subjects (Odom, 1972; Odom \& Lemond, 1975). In this research, the relevant and incidental stimulus components were presented within single compound stimuli (e.g., colored shapes), whereas, in the studies reviewed by Hagen and Hale (1973), the component values of the relevant and incidental dimensions were usually separated spatially. Hale and Piper (1973), however, included a compound stimulus condition and found more incidental learning in older than in younger subjects, replicating the results of other research using compound stimuli. Since an increase in

The authors wish to express their appreciation to the children and staff of Una Elementary School for their participation and assistance in the study. Requests for reprints should be sent to Richard D. Odom, Department of Psychology, Vanderbilt University, Nashville, Tennessee 37240. incidental recall with age is not readily accounted for by their selective-attention position, Hagen and Hale (1973) proposed that it may be more efficient for the older subjects to process component values of both relevant and incidental dimensions when those values are presented in a compound stimulus because "considerable effort must be expended in the initial discrimination process" (p. 126). The effort expended presumably is in determining which component of the stimulus compound is relevant and should be attended to and which component is irrelevant and should be ignored.

Inherent in this interpretation, however, is the problem of determining how the development of attentional processes should be measured. Hagen and Hale (1973) have proposed that a decrease in incidental recall with age would reflect improved efficiency in selective attention. It therefore would seem to follow that an increase in incidental recall with age would reflect a decreasing efficiency in selective processes. To be consistent within this position, the finding that older subjects had better incidental recall than younger subjects would then have to be interpreted as indicating that the older subjects had greater difficulty ignoring the incidental information than did the younger subjects.

Problems of this type may be avoided if the account of the age-related performance associated with both the compound- and component-stimulus conditions includes consideration of the perceptual characteristics of the task information and how they might be determined by the development of the perceptual system. Odom and Guzman (1972) have characterized one aspect of perceptual organization as a hierarchy of differential sensitivity to relations (e.g., categories or dimensions). Hierarchies comprised of a given set of categories and dimensions have been assessed and demonstrated using 
traditional salience tasks. Furthermore, research in which the salience of categories was assessed prior to their use in problems has shown that salience determines the likelihood that those categories will be cognitively evaluated for problem solution. That is, the higher the salience of a particular dimension, the higher the probability of its being evaluated and analyzed regardless of its appropriateness for problem solution. Because amount of exposure to all kinds of information through perceptual experiences of seeing, hearing, etc. normally increases with age, older subjects would be expected to be more perceptually sensitive in an absolute sense to more information and, consequently, would be expected to evaluate more of the available information in a given problem than younger subjects. In studies by Odom (1972) and Odom and Lemond (1975), the salience of the relevant and incidental dimensions was positively related to recall of values of both dimensions. Even though overall recall of incidental information increased with age, subjects at one age level showed more or less incidental recall of values from a particular dimension than those at another age level as a function of the salience of the dimension being recalled. For example, kindergarten subjects showed less incidental recall of values from a dimension low in salience than sixth-grade subjects showed with values from a dimension high in salience. Such results were found even though values from all dimensions were treated equivalently in the task procedure; that is, the requirements for problem solution were the same in all tasks. These findings reflect the influence of the perceptual characteristics of the information and are incompatible with the notion that the ability to ignore incidental information increases with age.

Another difference among the studies discussed above is in the specificity of the task instructions. In the studies reviewed by Hagen and Hale (1973), subjects were instructed to remember only the relevant dimensional values, whereas subjects in the Odom (1972) and Odom and Lemond (1975) studies were probed for the array locations of such values without prior identification of the relevant dimension. The manipulation of instructions to induce a cognitive set for evaluating only the relevant information in a task where the perceptual salience of the information is also manipulated should provide information about the independent and interactive roles of perception and cognition. This was assessed in the present study by presenting two age groups with a recall problem in which perceptual salience and instructions about the relevant dimension were varied.

\section{METHOD}

\section{Subjects and Salience Assessment}

The subjects were 32 kindergarten (mean age 5 years 11 months) and 32 sixth-grade (mean age 12 years) middle-class children from a Nashville public school. Three weeks before participation in the problem-solving task, the relative salience of the dimensions of form, color, and position was assessed for each subject. The assessment task was a modified method of triads, similar to the one used by Odom and Guzman (1972). The task consisted of nine items, each consisting of a single black card on which three $11.5 \times 11.5 \mathrm{~cm}$ white cards were located. On each white card were compounds of values from the three dimensions. The colors were yellow, blue, red, green, purple, and orange. The forms included triangle, square, cross, pentagon, circle, and inverted " $T$ "; position was represented by stimuli on each card being placed either across the vertical midline, along the upper horizontal edge, along the upper right to lower left diagonal, across the horizontal midline, along the right vertical edge, or along the upper left to lower right diagonal. There were two different sets of nine items, one using the first three values of each dimension listed above and the other using the remaining values. Each set was assigned to half of the subjects at each age level.

Each item provided a choice between two pairs of cards. One pair had identical values from one dimension, and the second pair had identical values from another dimension. The three cards on every item each contained a value which was different from the third dimension. The subject was instructed to choose the pair on each item that was most alike. Using the frequency of choice score for each dimension, an $\mathrm{ABC}$ hierarchy was calculated for each subject, with A designating the most salient (most frequently chosen) and $\mathrm{C}$ the least salient dimension.

\section{Recall Tasks}

Materials. The stimulus array was presented to each subject in a $82.6 \times 30.3 \mathrm{~cm}$ sloping tray, which was divided into six $17.8 \times$ $12.7 \mathrm{~cm}$ compartments. The tray was covered by a hinged lid. Six $12.7 \times 12.7 \mathrm{~cm}$ blank white cards were placed on the outside of the lid in a row corresponding to the tray compartments. In each tray compartment was one stimulus card containing a relevant dimensional value located above or below a second stimulus card containing an incidental dimensional value. The above-below location of the relevant and incidental stimulus cards was alternated randomly across the six compartments, with the limitation that neither occurred in the same location in more than two adjacent compartments. Two stimulus arrays with card locations and pairings were used and counterbalanced across subjects.

There were 18 white stimulus cards, each of which contained one of the 18 dimensional values used in salience assessment. In the center of each form card was pasted a black cutout of one of the six form values. In the center of each card representing color, an asymmetrical cutout of one of the six color values was attached. On each of the remaining six cards, there was a black rectangular strip corresponding to one of the position values. An identical set of 18 cards was used as probes for recall.

Procedure. Half of the subjects in each age group were randomly assigned to Condition AC (A dimension relevant and C dimension incidental). The remaining subjects were assigned to Condition CA ( $\mathrm{C}$ dimension relevant, A dimension incidental).

Each subject faced the apparatus and sat across the table from an experimenter who had not been involved in the earlier assessment of salience. Before a session began, the 12 stimulus cards representing the two dimensions appropriate for each subject were already arranged under the closed lid of the apparatus. There were 12 probe cards, identical to those in the covered array, located in front of the apparatus. Those from the relevant dimension were arranged in two rows of three cards each on a blank sheet of cardboard, located to the subject's left. The six incidental probe cards were arranged in the same fashion and located to the subject's right. Both sets of cards were positioned in one of two predetermined orders which were counterbalanced across subjects.

Within the salience conditions, half of the subjects were given set instructions in addition to the basic instructions which all subjects received. For the instructions given to subjects in all conditions, the experimenter gestured across the probe cards in front of the apparatus and told subjects that they would see identical cards under the lid. They were then told that they should remember where the cards under the lid were located 
because they would be asked to point to the places on the lid which those cards were under. For subjects receiving only these instructions, all of the probe cards were then simultaneously removed from in front of the apparatus without any designation about which set would be relevant. For subjects receiving set instructions, only the probe cards representing the incidental dimension were removed, and the subjects were told that the remaining probe cards were the important ones and that their locations under the lid should be remembered.

Immediately after the probe cards were removed, the initial 24-trial recall task began. Each trial began with a 5-sec exposure of the stimulus array. The experimenter then showed the subject a probe card with a value from the relevant dimension and asked the subject to point to the lid location which was occupied by that value in the array. Each of the six values was probed once during each of four blocks of six trials. If a subject made an error during the first six trials, he was corrected and shown the lid location. Values were probed according to one of two presentation orders which were counterbalanced across subjects and which differed from the left-to-right location of values in the stimulus array.

Following the initial task, six probes were given for the incidental dimension. The procedure was identical to that of the initial task, except that the stimulus array was not exposed and no feedback was provided.

\section{RESULTS}

\section{Initial Task}

The mean number of errors made by the age groups in the different conditions are presented in Table 1. A 2 (grade) by 2 (salience condition) by 2 (type of instruction) by 4 (trial block) analysis of variance on the number of errors in the initial task revealed a significant Grade effect $[F(1,56)=19.22, p<.001]$, with sixth graders making fewer total errors $(M=9.31)$ than kindergartners $(M=13.6)$. Salience condition was also significant $[F(1,56)=9.24, p<.004]$, there being fewer errors when the more salient dimension was relevant for task solution (Condition $\mathrm{AC}, \mathrm{M}=9.97$ ) than when the less salient dimension was relevant (Condition CA, M = 12.94).

As Table 1 shows, errors decreased significantly across trial blocks $[F(3,168)=26.02, p<.001]$. The significant Salience Condition by Trial Block interaction $[\mathrm{F}(3,168)=4.34, p<.006]$ reflected the greater decrease in errors across trial blocks in Condition AC (62\% decrease) than in Condition CA (25\% decrease). Type of instruction did not affect errors $(F=1.72)$, and no reliable differences were obtained for any of the seven interactions with which it was associated (all Fs $<1.00$ ).

Table 1

Mean Errors Made in Recalling Values of the Relevant Dimension in the Initial Problem

\begin{tabular}{|c|c|c|c|c|c|c|}
\hline \multirow[b]{2}{*}{ Grade } & \multirow{2}{*}{$\begin{array}{c}\text { Salience } \\
\text { Con- } \\
\text { dition }\end{array}$} & \multicolumn{4}{|c|}{ Trial Blocks } & \multirow{2}{*}{$\begin{array}{l}\text { Com- } \\
\text { bined }\end{array}$} \\
\hline & & 1 & 2 & 3 & 4 & \\
\hline Kindergarten & $\begin{array}{l}\mathrm{AC} \\
\mathrm{CA}\end{array}$ & $\begin{array}{l}4.06 \\
4.38\end{array}$ & $\begin{array}{l}3.00 \\
4.00\end{array}$ & $\begin{array}{l}2.88 \\
3.69\end{array}$ & $\begin{array}{l}1.81 \\
3.38\end{array}$ & $\begin{array}{l}11.75 \\
15.45\end{array}$ \\
\hline Sixth & $\begin{array}{l}\mathrm{AC} \\
\mathrm{CA}\end{array}$ & $\begin{array}{l}3.50 \\
3.19\end{array}$ & $\begin{array}{l}2.19 \\
2.81\end{array}$ & $\begin{array}{l}1.44 \\
2.13\end{array}$ & $\begin{array}{l}1.06 \\
2.31\end{array}$ & $\begin{array}{r}8.19 \\
10.44\end{array}$ \\
\hline Combined & & 3.78 & 3.00 & 2.54 & 2.14 & \\
\hline
\end{tabular}

\section{Incidental Task}

A 2 (grade) by 2 (salience condition) by 2 (type of instruction) analysis of variance was performed on the number of incidental recall errors. The Grade effect was significant at less than the .053 level $[\mathrm{F}(1,56)=3.82]$, with sixth graders making fewer errors $(M=3.78)$ than kindergartners $(M=4.41)$. Salience condition was also significant $[F(1,56)=15.26, p<.001]$, with fewer errors being made when the incidental dimension was high in salience $(M=3.47)$ than when it was low in salience $(M=4.72)$. Type of instruction was not a significant factor $(F<1.00)$, nor did it interact with either of the other variables to affect recall significantly (largest $F=3.09$ for the three-way interaction, $\mathrm{df}=1 / 56)$.

To ascertain whether incidental recall was significantly better than chance, $t$ tests were performed to compare the mean errors made by subjects in each of four grade by salience condition groups against chance level alone $(M=5.0)$. The only subjects who did not make significantly fewer errors than would be expected by chance-and who consequently showed no evidence of incidental recall-were the kindergartners in Condition $\mathrm{AC}(\mathrm{M}=5.13) .^{1}$

\section{DISCUSSION}

As demonstrated in other studies (Odom, 1972; Odom \& Lemond, 1975), the current results indicate that perceptual salience affects the recall of both relevant and incidental information. The incidental recall data provide support for a perceptual salience position (e.g., Odom \& Guzman, 1972) in that both younger and older subjects recalled significantly more values from their more salient dimension (A) than from their less salient dimension (C), even though both dimensions were irrelevant to solving the problem. In the initial recall task, both the kindergartners and sixth graders were more accurate in recall with successive exposures to the array, indicating that they were solving the initial problem.

Further effects of salience on performance in the initial task may be reflected in the data of the fourth trial block. Table 1 shows that the younger subjects' performance in the AC condition, where the information was high in salience, was similar to that of the older subjects in both salience conditions.

The results are, therefore, not in accord with interpretations of the development of problem solving which emphasize changes in cognitive, selective processes. Hagen and Hale (1973) proposed that with age children become better able to focus their attention on relevant and to ignore incidental information. However, from their position, a comparison of the incidental recall of older and younger subjects in the present study indicates that older subjects were not better able to ignore incidental information. Older subjects' recall was significantly better than chance in both salience conditions, while the younger subjects in the AC condition performed at chance level, in other words, they showed no incidental recall. That the younger children were able to perceive the $C$ dimension and discriminate among its values is evidenced by their improved recall across the trials of the CA condition in the initial problem. However, the $\mathrm{C}$ dimension apparently was of such low salience for the younger subjects that they rarely recalled its values when the dimension was irrelevant to problem solution. These results suggest that the perceptual characteristics of problem-solving tasks merit careful attention because of their significant interaction with cognitive processes. As noted, certain age-related similarities in the recall of relevant information and certain 
age-related differences in the recall of incidental information occurred as a function of the perceptual salience of that information.

Furthermore, specifically telling subjects which dimension was relevant to problem solution did not improve recall of those values or weaken recall of values from the incidental dimension. For both age groups, the major determinant of the recall of both relevant and incidental information was perceptual salience.

In the present study, the values of the relevant and incidental dimensions were spatially separated, as they were in the studies reviewed by Hagen and Hale. Nevertheless, the results agree with those from studies using compound stimuli where salience was assessed and where performance in incidental recall improved with age (Odom, 1972; Odom \& Lemond, 1975). In the case of the studies reviewed by Hagen and Hale, had there been assessment and manipulation of the perceptual salience of the categories comprised of patterned stimuli (animals and household objects), the results of these studies might have also resembled those of studies with compound stimuli. Recent findings (Odom \& Lemond, 1974) indicate that categories of patterned stimuli (in this case facial expression and facial identity) may differ in salience. Furthermore, the salience hierarchies of such categories of patterned stimuli may be related to age, and thus should be considered in any interpretation of developmental differences in problem-solving performance.

The age differences for both relevant and incidental recall may reflect developmental differences in the absolute salience of dimensions and/or cognitive operations that determine how much information can be processed and remembered in a given period of time. Although absolute salience has been shown to play a role within age levels (Odom \& Corbin, 1973; Odom, Astor, \& Cunningham, 1975), the appropriateness of either a salience or cognitive-change account of age-related results in recall cannot be clearly determined by the present study or any other in the literature. Nevertheless, from this and other investigations of salience and problem solving, it seems clear that if we are to understand the development of the structure and function of cognitive processes that analyze, evaluate, and transform information, then the perceptual nature of that information and how it changes with development must also be understood.

\section{REFERENCES}

Hagen, J. W., \& Hale, G. A. The development of attention in children. In A. D. Pick (Ed.), Minnesota symposia on child psychology (Vol. 7). Minneapolis: University of Minnesota Press, 1973.

Hale, G. A., \& Piper, R. A. Developmental trends in children's incidental learning: Some critical stimulus differences. Developmental Psychology, 1973, 8, 327-335.

ODom, R. D. Effects of perceptual salience on the recall of relevant and incidental dimensional values: A developmental study. Journal of Experimental Psychology, 1972, 92, 285-291.

Onom, R. D., Astor, E. C., \& Cunningham, J. G. The effects of perceptual salience on the matrix task performance of four- and six-year-old children. Child Development, 1975, 46, 758-762.

Opom, R. D., \& Corbin, D. W. Perceptual salience and children's multi-dimensional problem solving. Child Development, 1973, 44, 425-432.

ODom, R. D., \& Guzman, R. D. Development of hierarchies of dimensional salience. Developmental Psychology, 1972, 6, 271-287.

ODOM, R. D., \& LEMond, C. M. Children's use of component patterns of faces in multidimensional recall problems. Child Development, 1974, 45, 527-531.

ODOM, R. D., \& LEMOND, C. M. The recall of relevant and incidental dimensional values as a function of perceptual salience, cognitive set, and age. Journal of Experimental Child Psychology, 1975, 19, 524-535.

\section{NOTE}

1. In much of the extant literature on incidental learning, it is difficult to determine whether claims for incidental recall, that is, for more than chance-level performance, are justified. If support for cognitive-change positions, for example, that of Hagen and Hale, is to be based on the occurrence of such recall, then it must be shown that incidental information was recalled at an above-chance level of accuracy.

(Received for publication August 19, 1976.) 